\title{
Is electric charge separation the main process for kinetic energy transformation into the meteor phenomenon?
}

\author{
P. Spurný and Z. Ceplecha
}

\begin{abstract}
Astronomical Institute of the Academy of Sciences v.v.i., Ondřejov Observatory, Czech Republic e-mail: spurny@asu.cas.cz
\end{abstract}

Received 26 April 2008 / Accepted 24 June 2008

\begin{abstract}
After 3 years of systematic monitoring of fireballs by autonomous fireball observatories (AFO), millisecond flares (spikes) were recognized for the frequent and regular behavior of meteor light curves recorded with high time resolution. Also, other peculiarities in meteor motion, radiation, and ionization point to some more powerful internal processes in meteoroid interaction with the atmosphere than hypersonic aerodynamics can offer. Fragmentation of meteoroids starts at much higher altitudes than at those corresponding to aerodynamic loading. The fragility of meteoroids cannot explain this behavior, beeing also observed with the strongest bolide types (I and II). We propose triboelectricity as induced in meteoroids during their atmospheric penetration as the main process to adequately explain the meteor phenomenon, with hypersonic aerodynamics processes being only of secondary importance. Triboelectric charging may be the most important energy transfer inside a meteoroid causing internal charge differences inside different conductivity domains until discharge fragments the body. Such a process then may repeat over and over in the main body, as well as in individual fragments.
\end{abstract}

Key words. meteors, meteoroids

\section{Introduction}

By the term meteor phenomenon we understand the visible radiation stemming from hypersonic velocity penetration of a meteoroid (meteoric body) through the atmosphere. The traditional explanation of kinetic energy transformation into meteoric radiation assumes heating followed by melting, evaporation, or direct sublimation transforming the body gradually into hot gas. Observations have shown that the luminous volume in the vicinity of a meteoroid is composed of hot gas only slightly ionized and radiating in rather low energetic levels of mostly neutral or singly ionized atoms and molecules. This classical view and hypersonic-aerodynamics theory cannot explain new and overwhelming features of meteor radiation, millisecond flares, which we have recently revealed by applying high time-resolution photometry inside the AFO systems. Almost all light curves of meteors in our representative sample contain millisecond flares, including the strongest bolide type-I events. We present several examples of the light curves characteristic for the newly revealed radiation pattern of meteor radiation. We then present a possible and completely new explanation of this powerful time change in the conversion of meteoroid kinetic energy into light. It is meant as rather very preliminary and qualitative explanation, a guidance for future research.

\section{Millisecond flares (spikes)}

Very short flares were first observed on the light curves of bolides observed by satellite sensors with high time resolution of records (Tagliafferi et al. 1994). At first they were assumed to be an exception. Spalding and Jacobs constructed radiometers with high time resolution and Spurný put them in a systematic observing schedule within the European Fireball Network (EN) (Spurný et al. 2001). Soon it became evident that such extremely short flares are more frequent than suspected. Spurný \& Borovička (2002) then constructed an autonomous fireball observatory (AFO) which (except all-sky photographic system) also contains a high time-resolution photometer based on using a photomultiplier tube. The AFO's are now in regular operation at 10 Czech stations and one Slovak station of the EN (Spurný et al. 2007) and at 4 stations of the new Desert Fireball Network (DN) in southwestern Australia (Bland et al. 2006).

After 3 years of systematic monitoring of fireballs by this new observing technique, we recorded more than one hundred fireballs with very precise atmospheric trajectories and orbital data and simultaneously with very detailed high time-resolution light curves. Examples of these AFO's light curves of three EN fireballs are shown in Figs. 1a,b, 2, and 3. Figures 1a and $\mathrm{b}$ demonstrate the excellent fidelity of these records taken independently by two different AFOs from two different locations. It is evident that even small details in both records of the EN170907 fireball correspond very well and are thus real light-intensity fluctuations. The average noise at both stations is about 15 ( $y$-axis is in a linear scale and arbitrary units) and thus the resulting maximum signal-to-noise ratio is more than 100 at both stations. Such a high signal-to-noice ratio is typical of the majority of photographically recorded fireballs, i.e. of the fireballs with complete datasets. Similarly the radiometric light curves are available from at least two stations for the vast majority of recorded fireballs. Figure 2 shows the light curve of the fireball EN231006A, which is one of the best documented fireballs in terms of radiometric recordings. This fireball was bright enough to be recorded with reasonable signal-to-noice $(>100)$ at 5 Czech stations of the EN and the consistency of all records is perfect. Each (even tiny) flare is real including very fast fluctuations visible near the end on the presented light curve. This fireball belonged to the type I (the strongest meteoroids) with 

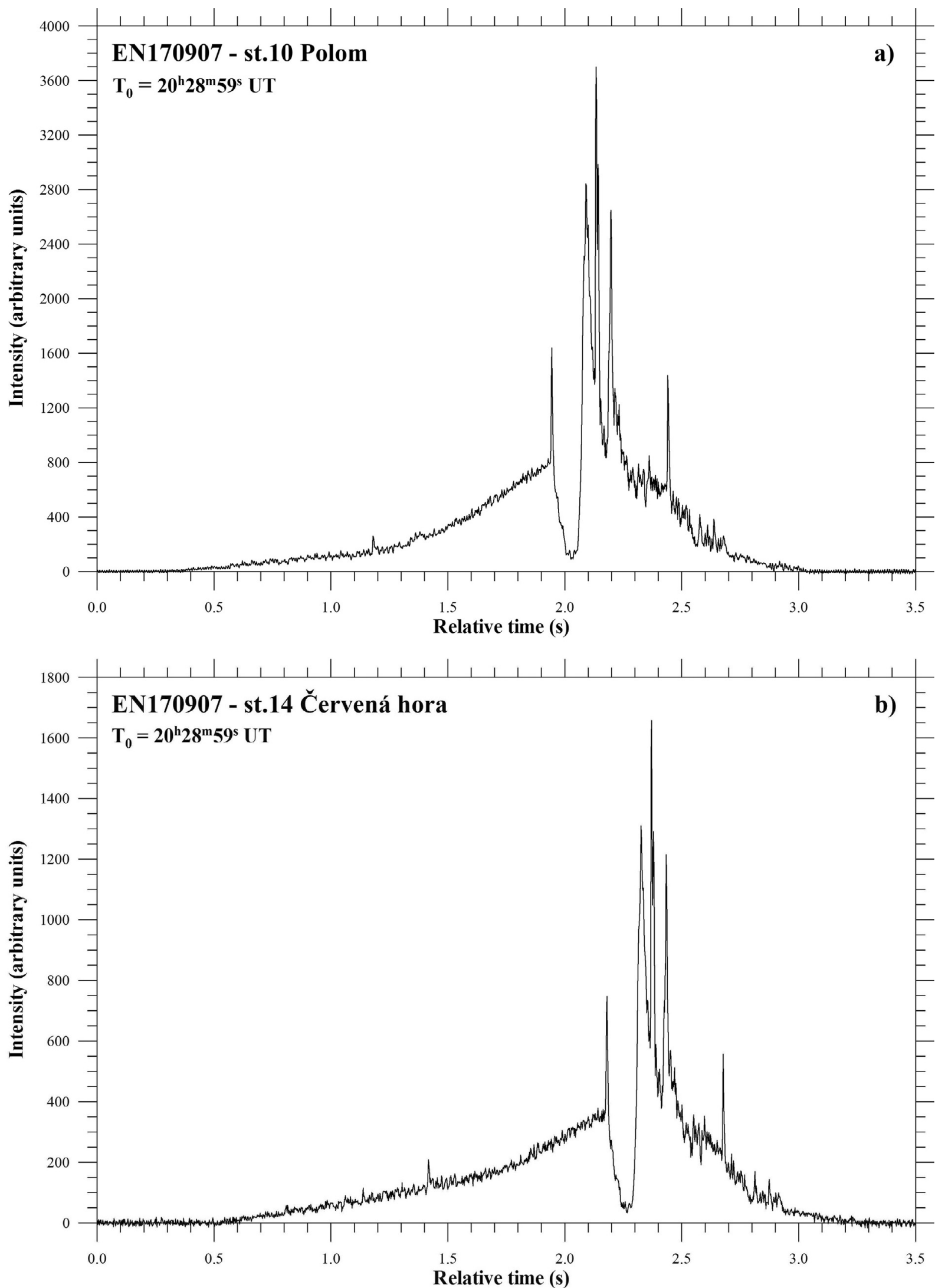

Fig. 1. Radiometric light curves of the EN170907 type II fireball recorded at the Czech stations of the EN 10 Polom and 14 Červená hora. The excellent fidelity of both records is clearly shown. The peculiarity of this fireball is in relatively long significant sag of brightness near the middle of luminous trajectory. 
P. Spurný and Z. Ceplecha: Is charge separation the main process for energy transformation into the meteor phenomenon?

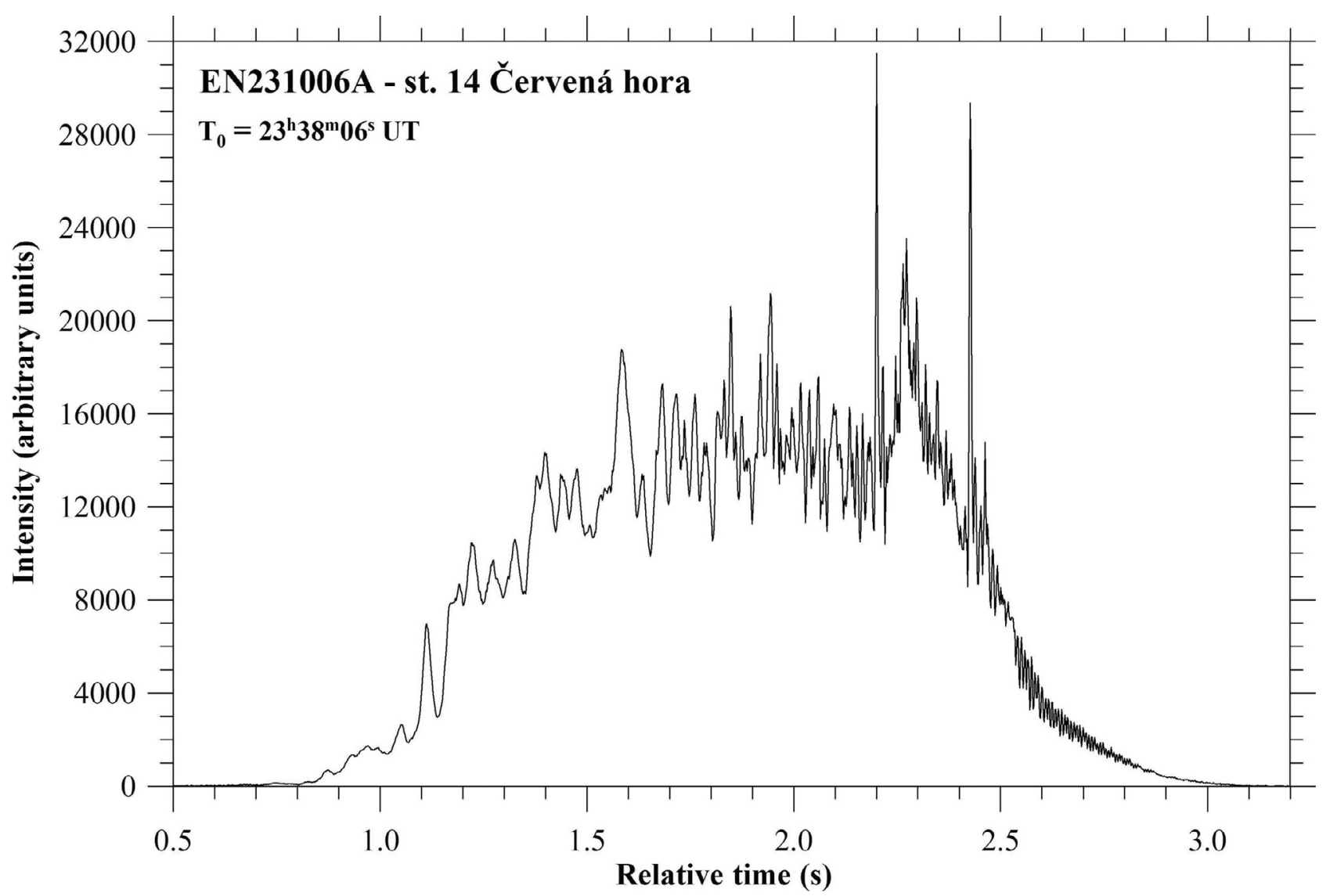

Fig. 2. Radiometric light curve of the EN231006A type I fireball from the Czech station Červená hora.

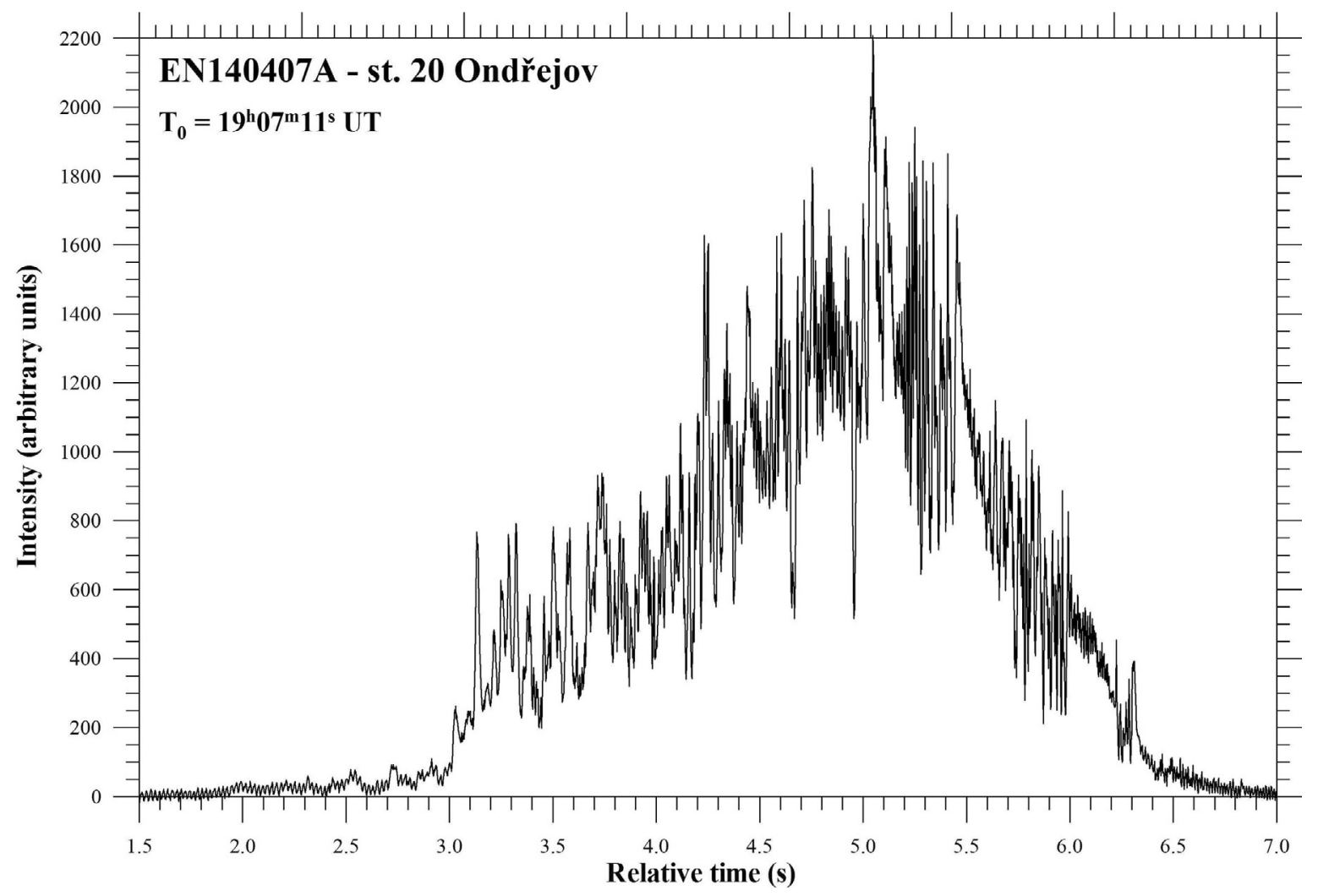

Fig. 3. Radiometric light curve of the EN140407A type I fireball from the Czech station Ondřejov. 
non negligible terminal mass on the order of $100 \mathrm{~g}$ and a fairly high initial velocity of $29 \mathrm{~km}$.

The light curve of this fireball documents that very short, millisecond flares are common events on almost all (at least 90\%) fireballs which we recorded by this new high time-resolution photometers. This statement is based on a very representative sample containing more than 100 fireballs. There is no significant preference by these very short flares for fireballs belonging toward some particular type or origin anticipated from their atmospheric behavior and orbits (asteroidal or cometary). However type I fireballs (presumed to have an asteroidal origin) have generally a much higher rate of millisecond flares (as shown in Fig. 3) than the types of weaker fireballs. The millisecond flares were thus recognized as a frequent and regular phenomenon for most of the recorded high-resolution lightcurves of fireballs. Discovering this phenomenon could not have been possible before, because meteors recorded by the regular photographic technique did not show such short flares smeared by consecutively superposing images. Similarly, TV records did not have enough time resolution to discover this common event. Now we have enough high time-resolution records to draw some preliminary statements thereby completely changing our understanding of meteor phenomenon.

\section{Meteor motion, radiation, and ionization pecularities}

The order difference of dynamically and photometrically determined masses of the same meteoroid formed a long lasting enigma in meteor physics. This problem was recently resolved by Ceplecha and ReVelle (2005) presenting a proper inclusion of fragmentation process into a model of meteoroid atmospheric penetration. Only apparent values (in contrast to a priori unknown intrinsic values) of the ablation coefficient, as well as of the luminous efficiency, can be determined when fragmentation is not properly included in a model of meteoroid atmospheric flight. The intrinsic values of the ablation coefficient and of the luminous efficiency are then those values resulting from the application of the fragmentation model. All well and precisely observed meteoric events (double- and multi-station photographs) studied by the fragmentation model so far, have yielded good results by accurately fitting motion and radiation of the examined meteoroids in the atmosphere. The model of Ceplecha \& ReVelle (2005) assumes fragmentation of a meteoroid as existing, however, it does not deal with the reasons for it. Why are meteoroids, generally speaking, so fragile in the atmosphere? Do they have that different a structure compared to all materials we know in our solar system from meteorites collections, sample returns, and in situ investigations? In this paper we try to outline the possible physics of the fragmentation process without proposing details.

The investigation of meteor motion itself (without taking the light emission into account) was presented in a paper by Ceplecha et al. (1993). Because only larger fragments can be studied this way, we call this type of fragmentation simply gross-fragmentation. The cited paper made use of photographic double- and multi-station records of fireballs. Grossfragmentation was observed either directly (splitting trajectories) or indirectly (sudden velocity changes of the main body). Very low pressures, at which the gross-fragmentation took place, was one of the main results of the paper. Most fragmentation of strong meteoric material (type I and II meteoroids) occurred between 0.1 and 1.2 MPa, i.e., more than one order less dynamic pressure than the strengths of materials of recovered meteoric stones (meteorites). The side velocities of fragments (velocity component perpendicular to the main-body trajectory) were found to be on the order of $1 \mathrm{~km} \mathrm{~s}^{-1}$. The first high side-velocity $(720 \mathrm{~m} / \mathrm{s})$ of a meteoroid fragment after breakage (and flare) at a height of $63 \mathrm{~km}$ was recognized by Ceplecha (1953) from double-station photographic record of a Southern Taurid. Inner gas overpressure (originally imbedded or chemically induced) was proposed as an explanation. Collisions in space causing the highly shocked structure found in some meteorites seem a highly improbable explanation for all events studied in Ceplecha et al. (1993). Moreover, collisions in space do not explain the high side velocities. It looks like the meteoroids were loaded by some unknown explosive.

Very precise photographic observations of 22 meteoroids (with standard deviations better than $15 \mathrm{~m}$ of observed against computed distances along the trajectory) were used to derive mass-loss and shape-density coefficients as functions of time (Ceplecha et al. 2000). These were the events for which the gross-fragmentation solution (with constant ablation and shapedensity coefficient) was impossible. Almost all examined cases (20) show much higher values (up to 50 times higher) of deceleration in the early parts of their luminous trajectories than corresponds to the instantaneous air density, thus implying much higher shape-density coefficients than physically feasible. Deviation in the air density from the monthly CIRA model (used in that paper) at heights of $70 \mathrm{~km}$ by a factor of 10 over a height difference on the order of $10 \mathrm{~km}$ is highly improbable. Very flat shapes of the meteoroids (flatter than a coin) or bulk densities that would be extremely low and increasing with time are also two very improbable explanations. A change of the average bulk density of a meteoroid by a factor of 10 during $1 \mathrm{~s}$ seems highly improbable. Also events were found with acceleration during an extensive early part of their trajectories. These events cannot be explained by meteor-physics equations at all. Some substantial unrecognized force had to act upon the motion of those meteoroids.

Also during lower parts of atmospheric trajectories of large meteoroids followed by meteorite falls, fragmentation is a powerful process as documented by well-observed video-recorded events (Peekskill: Brown et al. 1994; Morávka: Borovička \& Kalenda 2003). Side velocities at fragmentation points had higher values than aerodynamic forces can induce. Moreover, Borovička and Kalenda (2003) observed a completely new type of fragmentation they called sparks: fragments moving against motion of the source meteoroid fragment with velocities of about $8 \mathrm{~km} \mathrm{~s}^{-1}$ for a very short instant. The extremely detailed study of the fragmentation process of the Morávka meteoroid led to a conclusion that the dispersion of the fragments was significant in a cascade of consecutive fragmentation events. Some pieces achieved lateral velocities of up to $300 \mathrm{~m} / \mathrm{s}$, an order of magnitude more than could be explained by aerodynamic loading. An internal source for these energetic explosions should be sought.

Similar events have also been observed for larger highvelocity cometary meteoroids, especially for Leonids. Spurný et al. (2000) described jet-like structures detectable several kilometers sideways from the brightest parts of meteor head, and moving with a velocity over $100 \mathrm{~km} \mathrm{~s}^{-1}$ for several bright Leonids observed at very high altitudes (above $130 \mathrm{~km}$ and up to $200 \mathrm{~km}$ ). Lower in the atmosphere and also for Leonids, similar unusual structures have been reported by Taylor et al. (2000) and LeBlanc et al. (2000). The jet-like structures perpendicular to the flight path with lengths up to $2 \mathrm{~km}$ were observed for several Leonids. They postulate that these unusual structures are caused by tiny meteoroid fragments (containing metallic grains) 
being rapidly ejected (side velocities $15-30 \mathrm{~km} \mathrm{~s}^{-1}$ ) away from the core meteoroid.

Millisecond flares (spikes) are typical of the light curves of a majority of fireballs as we presented in the preceding section. Their total energy (due to short duration) can be easily explained by the fragmentation model of Ceplecha \& ReVelle (2005); however, the power of the radiation is so extremely high that it cannot be explained by existing models at all. The amount of kinetic energy of a meteoroid and its fragments needed to convert into radiation is rather small and thus also a small amount of fragmented mass is enough to explain (Spurný \& Ceplecha 2005). However, the conversion of kinetic energy into light is so speedy and the power of the conversion so high that fragmentation and ablation in a hypersonic aerodynamic regime simply could not be the reason for the millisecond flares. It looks like some more powerful process is acting in these spikes.

Head echoes of Perseids observed by 2 MW Canadian radar yielded average velocities both lower and higher than the known (outside the atmosphere) velocities of the same Perseid meteoroids (Šimek et al. 1998). The initial velocity computed from these head echoes is the same as measured for Perseids by other techniques, i.e. about $60 \mathrm{~km} \mathrm{~s}^{-1}$. High accelerations (some values over $100 \mathrm{~km} / \mathrm{s}^{2}$ ) were determined for 33 events with high reliability (standard deviations not exceeding $10 \%$ of the values). It appears that the location of the ionization volume responsible for the head echo cannot be identical to the instantaneous meteoroid position. During regular radar observations at Ondrejov Observatory, an unusual echo appeared on November 16, 1994, which cannot be explained as meteoric (Šimek et al. 1999). The radial velocity of this echo was over $300 \mathrm{~km} \mathrm{~s}^{-1}$, moving away from the observing site (from a range of $170 \mathrm{~km}$ to $220 \mathrm{~km}$ ). Any malfunction of the facility or an artificially introduced artifact were excluded by the authors. They were not able to explain this radar echo by any known natural phenomenon.

\section{Electrical phenomena in meteors}

Video and photometric observations of a meteor-triggered "jet" event in association with the occurrence of a sprite were collected during the SPRITES '98 campaign by Los Alamos National Laboratory group (Suszcynsky et al. 1999). A moderately bright meteor was recorded at heights from 100 to $70 \mathrm{~km}$, followed by a sprite between 86 and $57 \mathrm{~km}$, and then followed by a jet in the meteor trajectory moving in opposite direction from 80 to $84 \mathrm{~km}$ height with a velocity of 70 to $130 \mathrm{~km} \mathrm{~s}^{-1}$. This velocity is much too slow to suggest luminosity produced by a pure propagation of current up a fully ionized channel, and it implies a more complicated generation mechanism (Symbalisty et al. 2000). The width of the jet was about 1 to $2 \mathrm{~km}$. The authors conclude that this jet of luminosity propagating back up the trajectory of the meteor is almost certainly associated with the presence of the meteor ionization trail. They are not so sure about triggering the sprite itself mainly due to the unknown mechanism of such a process, but a chance coincidence seems to be a very low probability.

\section{Triboelectricity: proposals for future research in meteor physics}

The main result of the preceding sections: hypersonic aerodynamics considered so far in meteor physics as a primary process explaining conversion of kinetic energy into light is only of secondary importance. We propose triboelectricity induced in meteoroids during their atmospheric penetration with hypersonic velocities as the main process needed adequately explain the meteor phenomena (including hypersonic aerodynamics as a secondary process). We were not able to find theoretical or experimental data on the tribo effects of solids moving with hypersonic velocities in gases, so we are not able to present any quantification so far. Our ideas about this process follow.

A meteoroid loses free electrons from its surface by the friction of the air molecules already very high in the atmosphere. Positive charge accumulates gradually on the surface and electrons from the inside of the body thus try to move to the surface. Because of the inhomogeneity of the electric properties of meteoroid material, free electrons are negatively charging all low conductivity domains inside the body. This is due to the tendency of electrons to go to the positively-charged meteoroid surface, the low conductivity domains forming the obstacle to doing so. The charge inside the meteoroid is building up until voltage against surface layers is so high that a discharge tries to equal the potentials again. This induces a short high- temperature pulse in the body evaporating a small inner portion of the meteoroid and thus causing a high pressure exceeding the strength of the meteoroid material. Fragmentation starts (eventually with high side velocities of fragments). Such a process can be simply called explosion of the solid meteoroid. Chemical reactions could be also involved in such a process. The main body and the fragments continue to move with hypersonic forward-velocities in their new trajectories and the described process can start again.

Ions in such a process are also leaving the body, however, with much less efficiency than electrons. This way the surface charge of a meteoroid is kept positive except for the short instants of discharges. Also the wake behind a meteoroid should be positively charged closer to the body and negatively charged far behind it. In any case the process is outside the equilibrium, and the number of ions and electrons in any small volume inside, on the surface, and outside the meteoroid (in the volume directly influenced by the meteoroid) are not equal. Only all ions and electrons taking part in an entire meteor phenomenon should be equal not considering very large atmospheric volume electric phenomena (ionosphere layers) with their own large-scale currents.

The final stage of all processes during meteoroid penetration through the atmosphere is evaporation (sublimation), because we see meteor phenomenon as the fairly low temperature radiation of only slightly ionized gas. Borovička (1994) found that meteor radiation can be explained as the radiation of two independent volumes with LTE temperatures of about $4500 \mathrm{~K}$ and $10000 \mathrm{~K}$. This may just be a first approximation resulting from the assumption of the LTE. Because neutral atoms, ions, and free electrons are far away from the LTE, temperature is an illusive concept and the two different temperature volumes may represent the highly unequilibrated conditions. Explanation of meteor spectra needs computation of populations of electrons at different levels of radiating atoms, of radiating ions, and of free electrons.

In our new concept the bolide types I, II, IIIA, and IIIB (and meteor types A, B, C, D) correspond to differences in the meteoroid structure and composition in the sense that they mostly reflect inner differences of the electric properties of the material. Also the strength categories (Ceplecha et al. 1998) fit well into our proposed triboelectric meteor model. The fragmentation phenomena observed for faint Super-Schmidt meteors, e.g terminal blending, also fit into the explanation of meteors by overwhelming triboelectric phenomena in a hypersonic aerodynamics regime. 


\section{Conclusions}

1. Flares of millisecond duration (spikes) are regular features for almost all light curves observed with high time resolution.

2. The process of converting kinetic energy into light is so speedy in spikes and the power of the conversion so high that fragmentation and ablation in hypersonic aerodynamic regime cannot be the cause of the millisecond flares. Hypersonic aerodynamics cannot explain this extreme change in the power output with time.

3. Fragmentation of meteoroids starts at much higher altitudes, where aerodynamic pressure is not strong enough (two or more orders weaker) to break the body.

4. Side velocities of large fragments are much higher than aerodynamic forces can induce.

5. Triboelectric charging may be the most important energy transfer inside a meteoroid, causing internal charge differences until discharge fragments the body.

6. Charge inside a meteoroid builds up until voltage against surface layers is so high that a discharge tries to equal the potentials. This induces a short high-temperature pulse in the body, evaporating a small inner portion of the meteoroid, and in turn causing thus high pressure exceeding the strength of the meteoroid material and triggering fragmentation.

7. Aerodynamic forces are of secondary importance in the fragmentation of meteoroids.

8. Bolide types I, II, IIIA, IIIB and meteor types A, B, C, D may instead reflect inner differences in the electric properties of the meteoric material.

Acknowledgements. The authors are indebted to Dr. J. Borovička for his valuable comments. We also gratefully acknowledge helpful comments by the reviewer Dr. D. Koschny. This work was supported by GA ČR grant 205/08/0411 and the EU grant MRTN-CT-2006-035519. The institutional research plan number is AV0Z10030501.

\section{References}

Bland, P. A., Spurný, P., Bevan, A. W. R., et al. 2006, Meteorit, \& Planet. Sci., 41, 5197

Borovička, J. 1994, Planet. Space Sci., 42, 145

Borovička, J., \& Spurný, P. 1996, Icarus, 121, 484

Borovička, J., \& Kalenda, P. 2003, Meteorit. Planet. Sci., 38, 1023

Brown, P., Ceplecha, Z., Hawkes, R. L., et al. 1994, Nature, 367, 624

Ceplecha, Z. 1953, Bull. Astron. Inst. Czechosl., 4, 113

Ceplecha, Z., \& ReVelle, D. O. 2005, Meteorit, \& Planet. Sci., 40, 35

Ceplecha, Z., Spurný, P., Borovička, J., \& Keclíková, J. 1993, A\&A, 279, 615

Ceplecha, Z., Borovička, J., Elford, W.G., et al. 1998, Space Sci. Rev., 84, 327

Ceplecha, Z., Borovička, J., \& Spurný, P. 2000, A\&A, 357, 1115

LeBlanc, A. G., Murray, I. S., Hawkes, R. L., et al. 2000, MNRAS, 313, L9

Spurný, P., \& Borovička, J. 2002, in Proc. ACM 2002, ed. B. Warmbein, ESA SP-500, 257

Spurný, P., \& Ceplecha, Z., 2005, Earth, Moon, \& Planets, 95, 477

Spurný, P., Betlem, H., Jobse, K., Koten, P., \& van’t Leven, J. 2000, Meteorit, \& Planet. Sci., 35, 1109

Spurný, P., Spalding, R. E., \& Jacobs, C., 2001 in Proc. Meteoroids 2001 Conf., ed. B. Warmbein, ESA SP-495, 135

Spurný, P., Borovička, J., \& Shrbený, L., 2007, in Near Earth Objects, our Celestial Neighbors: Opportunity and Risk, ed. G. B. Valsecchi, \& D. Vokrouhlický, IAU Symp., 236, 121

Suszcynsky, D. M., Strabley, R., Roussel-Dupre, R., et al. 1999, J. Geophys. Res. Atmosph., 104, 31361

Symbalisty, E. M. D., Roussel-Dupre, R., ReVelle, D. O., et al. 2000, Icarus, 148, 65

Taylor, M. J., Gardner, L. C., Murray, I. S., P., \& Jenniskens, P. 2000, Earth, Moon \& Planets, 82-83, 379

Šimek, M., Přidal, P., Pecina, P., \& Boček, J. 1998, Earth, Moon \& Planets, 77, 1

Šimek, M., Pecina, P., \& Přidal, P. 1999, in Proc. Meteoroids 1998 Conf., ed. W. J. Baggaley, \& V. Porubčan, Polygrafia SAV, Bratislava, 98

Tagliaferri, E., Spalding, R., Jacobs, C, Worden, S. P., \& Erlich, A. 1994, in Hazards Due to Comets and Asteroids, ed. T. Gehrels (University of Arizona Press, Tucson), 199 